\title{
A theory of the epidemiology of gonorrhoea
}

\author{
W. F. FELTON \\ From the Royal Sussex County Hospital, Brighton, Sussex
}

SUMMARY Gonorrhoea is a typical endemic disease. Its continuity has been traditionally attributed to the existence of a pool of infected promiscuous women. Alternative theories have suggested a dynamic approach which gives equal importance to the man and the woman. Models constructed to simulate the epidemiology of gonorrhoea have so far failed to be realistic. An hypothesis that the distribution of infections is limited by the number of opportunities for transmission presented by the pattern of sexual behaviour may explain the low prevalence of the disease in England.

\section{Introduction}

Gonorrhoea is an endemic disease which is distinguished by its continuity from year to year and from century to century. Before and after the introduction of penicillin sudden and unexpected local outbreaks of infection have been rare. The Chief Medical Officer's statistics from 1918 until the present time (Ministry of Health, 1918-63; Department of Health and Social Security, 1964-77) have shown upward and downward trends, but either way the trends have lasted a decade or longer. There have been no sporadic or erratic changes of direction. The annual variation in the number of cases recorded has been of the order of $10 \%$ except around the wartime peaks. The upward trend of the last two decades is slowing. Nevertheless, the apparent failure to influence the distribution of gonorrhoea to any marked degree by successful treatment and by diligent contact-tracing calls for an explanation.

There is a notable lack of epidemiological data and much of what are available-for example, the Chief Medical Officer's figures-have been regarded as unsatisfactory. The Chief Medical Officer's figures, however, do cover a continuous period of 60 years, during which they have shown a remarkable consistency. They would seem to provide a worthwhile base on which to continue the theoretical discussion begun by Colonel Harrison (1917), who pioneered the organisation of clinics and the collection of their statistics.

INFECTIOUS PROMISCUOUS FEMALE POOL The difficulty of bringing contacts to diagnosis and treatment has beset clinicians since serious attempts

Address for reprints: Dr W. F. Felton, Royal Sussex County Hospital, Brighton BN2 5BE

Received for publication 29 May 1978 were made to bring gonorrhoea under control. Harrison (Ministry of Health, 1927) tried to measure this failure of discovery by comparing the relative incidence of syphilis and gonorrhoea in the armed Forces, where notification was compulsory, and in the civilian population, where it was not. In 1920 there were seven to 10 cases of gonorrhoea among Army, Navy, and Airforce personnel to one of syphilis, whereas among civilians the ratio of cases was more equally balanced at $2 \cdot 5: 1$ for men and 1.4:1 for women (Ministry of Health, 1927). This was an unfortunate comparison, since 1920 was a record year for syphilis with no less than 42805 cases. It is also clear now that the epidemiology of syphilis and gonorrhoea are not comparable, for the former has declined while the latter has increased in the same population.

In 1976 (Department of Health and Social Security, 1978) there were 3878 cases of syphilis and 58725 cases of gonorrhoea in England. This is a ratio of $1: 15$.

The idea of failure to discover cases was further supported by the imbalance of men and women which in 1920 was 5.2:1 and until 1961 remained at nearly $4: 1$. This suggested that the failure mainly concerned women, and, as it is well known, a large proportion of these are asymptomatic. The male-tofemale ratio for syphilis of 2.5:1 suggested that the gonorrhoea ratio would be lower if all cases were found and recorded. Harrison argued from this that there might have been more than 100000 cases in the population in 1920 of which less than half were brought to treatment (Ministry of Health, 1927).

In 1976 the male-to-female ratio for gonorrhoea was $1 \cdot 7: 1$ and for syphilis $3 \cdot 2: 1$. The position has practically reversed itself since 1920 . The change in the figure for syphilis is probably partly a reflection 
of the greater involvement of male homosexuals. It can also be observed with hindsight that the maleto-female ratio remained constant at near $4: 1$ for 40 years. It seems hardly likely that all the efforts at contact-tracing and a revolution in treatment following the use of penicillin would have failed to make some impression. If the existence of the imbalance was mainly due to a failure to discover female cases a change might have been expected before 1961 .

Today's authorities, still under the influence of this early theory, continue to write of an infectious female pool as though it is the key factor in the continuity of gonorrhoea (World Health Organisation, 1975). The basis for its existence has always been largely intuitive. It is perhaps more reasonable to suppose that the imbalance may be part of the natural history of the disease. There may always have been more male than female cases. If the mean infectious period in women is longer than that in men-a reasonable clinical proposition (Morton, 1972)-it is probable that women will infect more men than vice versa. If more men change partners more often than women, then it would follow that men are at greater risk and would acquire more infections.

There is no doubt that a reservoir of infectious men and women exists. In England the infectious female pool almost certainly does not consist of a group of stubbornly undiscoverable women. Willcox (1965a; 1966a, b, c; 1967), in the series of papers in which he develops his theory, points out that the pool is always losing and recruiting members. Recruitment is only possible with the aid of infectious men who are as important in the continuity of gonorrhoea as women. If this last point is taken, the analogy of the pool can be exchanged for that of a wave of infections in men and women passing through a population. The way is then open to propose a dynamic theory of gonorrhoea distribution.

\section{New theories}

Workers who have offered new ideas to explain endemic gonorrhoea have been confronted by a number of difficulties. The specialist epidemiologist looks for fields in which there is an existing or potential body of reliable data. It is clear that new data will be difficult and expensive to collect. Any new theories, like the old, will have to depend at first on existing data and clinical intuition.

Clinicians for their part have little time to read closely argued theoretical discussions which, when summarised, seem to offer little immediate hope of making their control task more effective. The sophisticated mathematical approach may leave the clinician without any clear understanding of what is proposed. On the other hand, the infectious pool theory is easily grasped and has the authority of longevity on its side. It offers a target to be found and destroyed.

Willcox (1965a) first developed the argument for a dynamic approach without resort to difficult mathematics. His model led him to postulate that gonorrhoea was balanced on a knife edge and may increase or decrease exponentially from a point of equilibrium. The continuity of both local and national trends is evidence against the existence of such a precarious situation.

Lee et al. (1972) devised a simple model which was based on the assumption that gonorrhoea had been increasing in the USA at an exponential rate. They reason that this means that there is a 'positive feedback loop' or that an increase now will lead to an even bigger increase later. From this they deduce that a relatively small reduction in transmissions of say $10 \%$ which might be achieved by the use of an intravaginal antiseptic compound could bring gonorrhoea under control.

Gladys Reynold's (1973) model, which has been used and elaborated by St John (1973), employs, from the clinician's point of view, difficult mathematics. She, like Lee et al. (1972), concludes that relatively small changes such as the reduction of the duration of infections in women from 100 to 92 days would halve the prevalence of gonorrhoea in about three years. If the reduction was maintained the prevalence would continue to fall.

In the light of the recorded experience of the last 60 years the clinician who doubts the validity of these propositions can hardly be called a cynic. Commonsense suggests that the world-wide use of penicillin was a major event probably reducing the mean duration of infections in women. In no country has the introduction of penicillin brought about a collapse of the endemic level. It is true that there was a false dawn in England during the early 1950s. Events now point strongly to the low figure of 1955 being due to social factors, such as the stabilisation of the pattern of sexual behaviour.

Two other models have been constructed, one by Beral (personal communication) and the other by Constable (1975). They both employ rather more difficult mathematics. They might both be described as second generation as they bring the endemic situation into equilibrium. This is a great advance, since the reason why an infection is endemic is that it is in a state of equilibrium within its host population. The incidence and prevalence are shown as relatively stable and not subject to wide fluctuations on account of small changes in the parameters of 
the models. The method of bringing about the equilibrium is, however, open to criticism and depends upon a sufficient number of infected men contacting infected women so that their respective infections are not reproduced. Clinical intuition finds this difficult to accept for a number of reasons, more especially because if it were true the seeking out and removal of infection might actually increase the incidence of disease.

The reason for casting doubt on the first two generations of new theory is to clear the ground for the third generation. The parameters must describe as faithfully as possible the pattern of sexual behaviour quantitatively in relation to time and space. On the microbiological side they must describe the characteristic infectious periods for the two sexes and the degrees of infectivity between the two sexes. It is well known, however, that there are few hard data to cover any of these matters and one reason for making a model is to try and fit some values into it. This experience can be helpful in the preparation of investigations and in determining priorities.

For the purpose of this part of the argument let us assume the following. Infectivity in either direction is of a high order, say $80 \%$. Natural or acquired immunity is an unimportant factor and if it exists is very rare. In England the great majority of cases of gonorrhoea are treated and the characteristic infectious period for men is around 10 days and for women about 30 days. The longstanding undiscovered case of either sex is the exception rather than the rule.

The dynamics of gonorrhoea are provided by the pattern of sexual behaviour and the movement of men and women within the population. This behaviour and movement provides the network through which the gonococcus passes from person to person and place to place. The network has to be in existence before an endemic situation can develop. An example is the spread that followed the introduction of gonorrhoea into the island of Tahiti by French and English sailors (Hunter, 1786).

The essential and basic element of the pattern of sexual behaviour is the paired partner change by one person. The first change provides the opportunity to acquire and the second to transmit infection. The paired partner change will only provide the opportunity for transmission if the interval between the changes is within the characteristic period of infectiousness. Thus what the gonococcus needs for its survival is a series of linked partner changes, all of which are correctly timed for transmission. In a population of tens of millions partner changes must occur by the thousand every day, but the probability that any one of those changes will be correctly linked in respect of time with a chain of other partner changes equally correctly linked is low. This explains why in a population of more than 45000000 in England the rate of discovery and eradication of gonorrhoea is limited to an average of 160 cases per day.

If the next generation of gonorrhoea models uses the limitation of opportunities for transmission as a means of demonstrating how infections are held in a position near to equilibrium the results will be more realistic. In particular, it will be possible to postulate a low rate of prevalence. The available evidence suggests that the prevalence in England is less than $1 \%$ (Silverstone et al., 1974).

It has already been proposed that the analogy of the pool should be replaced by that of a wave passing through the population in time. Its height is mainly determined by the number of opportunities presented to the gonococcus for its transmission. These opportunities are governed by social factors and are largely outside the influence of medical control programmes. The length of the wave is determined by the duration of infections. The length is also a function of the height, that is, the total number of infections. The duration of infections is within the scope of medical control programmes; indeed their main objective is to secure the earliest possible removal.

Unhappily even if the control programme is executed with both skill and enthusiasm the cases seen at the clinics are from the trailing edge of the wave. It has to be so because action is initiated on the discovery of an infection, which has often been present long enough to allow transmission. While it seems unlikely that it will ever be possible to control gonorrhoea satisfactorily, until infection can be blocked by providing artificial immunity, our present methods should be respected for the results they have achieved. The first achievement is the reduction of the complications to a level at which they are comparatively rare. The second is the reduction in the number of infections by shortening their duration. It seems likely that the fall in the male-to-female ratio may be partly the result of control. Thirdly, it is possible to speculate that the definitive diagnosis after screening at a clinic and the subsequent tracing of contacts slows downeven for a short time-the partner-change rate of the sexual grouping involved. This may result in the breaking of the chain of infection in the group. If this is true it is one way in which clinics are influencing what is happening at the leading edge of the wave. 
References

Constable, Gillian M. (1975). The problems of VD modelling. In the Proceedings of the 40th International Statistical Institute Conference, Warsaw 1975, Paper 19.

Department of Health and Social Security (1964-77). On the State of the Public Health: Annual Report of the Chief Medical Officer. HMSO: London.

Handsfield, H. H., Lipman, T. O., Harnisch, J. P., Tronca, E., and Holmes, K. K. (1974). Asymptomatic gonorrhoea in men. New England Journal of Medicine, 290, 117-123.

Harrison, L. W. (1917). The Diagnosis and Treatment of Venereal Disease in General Practice. Oxford Medical Publications: Oxford. Hunter, J. (1786). Treatise on the Venereal Disease. London.

Lee, T. Y., Utidjian, H. M. D., Singh, B., Carpenter, U., and Cutler, J. C. (1972). Potential impact of chemical prophylaxis on the incidence of gonorrhoea. British Journal of Venereal Diseases, 48, 376-379.

Ministry of Health (1918-63). On the State of the Public Health: Annual Report of the Chief Medical Officer. HMSO: London.

Morton, R. S. (1972). Venereal Diseases. Pelican Books: London.

Reynolds, Gladys H. (1973). A control model for gonorrhoea. An unpublished dissertation deposited in the library of Emory University, Atlanta, Georgia.
St John, R. K. (1973). Possible solutions to the problem of gonorrhoea. Early detection to prevent transmission. In Proceedings of the International Symposium on Gonorrhoea, Ottawa, Canada, 25 October, 1973.

Silverstone, P. I., Snodgrass, C. A., and Wigfield, A. S. (1974). Value of screening for gonorrhoea in obstetrics and gynaecology. British Journal of Venereal Diseases, 50, 53-56.

Willcox, R. R. (1965a). Importance of 'feedback' in gonorrhoea control. British Journal of Venereal Diseases, 41, 287-291.

Willcox, R. R. (1965b). The essence of gonorrhoea control. Acta dermato-venereologica, 45, 302-308.

Willcox, R. R. (1966a). The essence of gonorrhoea control. Acta dermato-venereologica, 46, 95-100.

Willcox, R. R. (1966b). The essence of gonorrhoea control. Acta dermato-venereologica, 46, 250-256.

Willcox, R. R. (1966c). The essence of gonorrhoea control. Acta dermato-venereologica, 46, 460-465.

Willcox, R. R. (1967). The essence of gonorrhoea control. Acta dermato-venereologica, 47, 65-69.

World Health Organisation (1975). Social and health aspects of sexually transmitted diseases: need for a better approach. A28/ Technical Discussions/1, p. 3, WHO: Geneva. 